\title{
Higher curvature quantum gravity and large extra dimensions
}

\author{
Durmuş A. Demir*, Şükrü H. Tanyıldızı \\ Department of Physics, Izmir Institute of Technology, IZTECH, TR-35430 Izmir, Turkey
}

Received 15 November 2005; received in revised form 26 November 2005; accepted 6 December 2005

Available online 13 December 2005

Editor: N. Glover

\begin{abstract}
We discuss effective interactions among brane matter induced by modifications of higher dimensional Einstein gravity via the replacement of Einstein-Hilbert term with a generic function $f(\mathcal{R})$ of the curvature scalar $\mathcal{R}$. After deriving the graviton propagator, we analyze impact of virtual graviton exchanges on particle interactions, and conclude that $f(\mathcal{R})$ gravity effects are best probed by high-energy processes involving massive gauge bosons, heavy fermions or the Higgs boson. We perform a comparative analysis of the predictions of $f(\mathcal{R})$ gravity and of Arkani-HamedDvali-Dimopoulos (ADD) scenario, and find that the former competes with the latter when $f^{\prime \prime}(0)$ is positive and comparable to the fundamental scale of gravity in higher dimensions. In addition, we briefly discuss graviton emission from the brane as well as its decays into brane-localized matter, and find that they hardly compete with the ADD expectations. Possible existence of higher-curvature gravitational interactions in large extra spatial dimensions opens up various signatures to be confronted with existing and future collider experiments.
\end{abstract}

(c) 2005 Elsevier B.V. All rights reserved.

\section{Introduction}

The relative feebleness of gravity with respect to the weak force and its stability under quantum fluctuations, the gauge hierarchy problem, has been pivotal for introducing a number of 'new physics' models to complete the standard electroweak theory (SM) above Fermi energies. The idea [1-3] that the scale of quantum gravity can be much lower than the Planck scale, possibly as low as the electroweak scale itself $[4,5]$ since this extreme is not excluded by the present experimental bounds [6], has opened up novel lines of thought and a number of phenomena which possess observable signatures in laboratory, astrophysical and cosmological environments.

The basic setup of the Arkani-Hamed-Dimopoulos-Dvali (ADD) scenario [5] is that $(1+3)$-dimensional universe we live in is a field-theoretic brane [7] which traps all flavors of matter except the SM singlets, e.g., the graviton and righthanded neutrinos. As long as the surface tension of the brane

\footnotetext{
* Corresponding author.

E-mail address: demir@physics.iztech.edu.tr (D.A. Demir).
}

does not exceed the fundamental scale $\bar{M}_{D}$ of $D$-dimensional gravity, at distances $\gg 1 / \bar{M}_{D}$ the spacetime metric $g_{A B}$ remains essentially flat. In other words, for singlet emissions (from brane) with transverse (to brane) momenta $\left|\vec{p}_{T}\right| \ll \bar{M}_{D}$ the background spacetime is basically Minkowski. Therefore, it is admissible to expand $D$-dimensional metric about a flat background

$g_{A B}=\eta_{A B}+2 \bar{M}_{D}^{1-D / 2} h_{A B}$,

where $\eta_{A B}=\operatorname{diag}(1,-1,-1, \ldots,-1)$ and $h_{A B}$ are perturbations. The gravitational sector is described by Einstein gravity

$S_{\mathrm{ADD}}=\int d^{D} x \sqrt{-g}\left\{-\frac{1}{2} \bar{M}_{D}^{D-2} \mathcal{R}+\mathcal{L}_{\text {matter }}\left(g_{A B}, \psi\right)\right\}$,

where $\psi$ collectively denotes the matter fields localized on the brane. There are various ways [5] to see that the Planck scale seen on the brane is related to the fundamental scale of gravity in higher dimensions via

$\bar{M}_{\mathrm{Pl}}=\sqrt{V_{\delta}} \bar{M}_{D}^{1+\delta / 2}$

which equals $(2 \pi R)^{1 / 2} \bar{M}_{D}^{1+\delta / 2}$ when $\delta \equiv D-4$ extra spatial dimensions are compactified over a torus of radius $R$. Obviously, 
larger the $R$ closer the $\bar{M}_{D}$ to the electroweak scale [5]. Experimentally, size of the extra dimensions, $R$, can be as large as a small fraction of millimeter [6], and thus, quantum gravitational effects can already show up at experimentally accessible energy domains provided that the strength of gravitational interactions on the brane drives from higher dimensional gravity as in (3). Upon compactification, the higher dimensional graviton gives rise to a tower of massive $S, P$ and $D$ states on the brane, and they participate in various scattering processes involving radiative corrections to SM parameters, missing energy signals as well as graviton exchange processes. These processes and their collider signatures have been discussed in detail in seminal papers [8,9].

The ADD mechanism is based on higher dimensional Einstein gravity with metric (1). Given the very fact that general covariance does not forbid the action density in (2) to be generalized to a generic function $f\left(\mathcal{R}, \square \mathcal{R}, \nabla_{A} \mathcal{R} \nabla^{A} \mathcal{R}, \mathcal{R}_{A B} \mathcal{R}^{A B}\right.$, $\left.\mathcal{R}_{A B C D} \mathcal{R}^{A B C D}, \ldots\right)$ of curvature invariants, in this work we will derive and analyze effective interactions among brane matter induced by such modifications of higher dimensional Einstein gravity, and compare them in strength and structure with those predicted by the ADD mechanism. The simplest generalization of (2) would be to consider, as we will do in what follows, a generic function $f(\mathcal{R})$ of the curvature scalar. Such modified gravity theories are known to be equivalent to Einstein gravity (with the same fundamental scale) plus a scalar field theory with the scalar field

$\phi=\bar{M}_{D}^{(D-2) / 2} \sqrt{\frac{D-1}{D-2}} \log \left|\frac{\partial f}{\partial R}\right|$

in a frame accessible by the conformal transformation $g_{A B} \rightarrow$ $(\partial f / \partial R) g_{A B}[10]$. Therefore, generalized action densities of the form $f(\mathcal{R})$ are equivalent to scalar-tensor theories of gravity, and thus, matter species are expected to experience an additional interaction due to the exchange of the scalar field $\phi$ [11]. This is the fundamental signature of $f(\mathcal{R})$ gravity compared to Einstein gravity for which simply $f(\mathcal{R})=\mathcal{R}$.

In this work we study how $f(\mathcal{R})$ gravity influences interactions among brane matter and certain collider processes to observe them. In Section 2 below we derive graviton propagator and describe how it interacts with brane matter. Here we put special emphasis on virtual graviton exchange. In Section 3 we study a number of higher dimensional operators which are sensitive to $f(\mathcal{R})$ gravity effects. In Section 4 we briefly discuss some further signatures of $f(\mathcal{R})$ gravity concerning graviton production and decay as well as certain loop observables on the brane. In Section 4 we conclude.

\section{Gravitational interactions from $f(\mathcal{R})$ gravity}

We parameterize generalized gravity theory via the action

$S=\int d^{D} x \sqrt{-g}\left\{-\frac{1}{2} \bar{M}_{D}^{D-2} f(\mathcal{R})+\mathcal{L}_{\text {matter }}\left(g_{A B}, \psi\right)\right\}$, where couplings to matter fields $\psi$ are identical to those in (2). The metric field obeys

$$
\begin{aligned}
& f^{\prime}(\mathcal{R}) \mathcal{R}_{A B}-\frac{1}{2} f(\mathcal{R}) g_{A B}+\left(g_{A B} \square-\nabla_{A} \nabla_{B}\right) f^{\prime}(\mathcal{R}) \\
& =-\frac{\mathcal{T}_{A B}}{\bar{M}_{D}^{D-2}},
\end{aligned}
$$

where prime denotes differentiation with respect to $\mathcal{R}$, and

$\mathcal{T}_{A B}=-\frac{2}{\sqrt{-g}} \frac{\delta\left(\sqrt{-g} \mathcal{L}_{\text {matter }}\right)}{\delta g^{A B}}=\delta^{\delta}(\vec{y}) \delta_{A}^{\mu} \delta_{B}^{\nu} T_{\mu \nu}(z)$

is the stress tensor of the brane matter where $y_{i}$ and $z_{\mu}$ stand, respectively, for coordinates in extra space and on the brane. The second equality here reflects the fact that entire energy and momentum are localized on the brane. Clearly, energy-momentum flow has to be conserved $\nabla^{A} \mathcal{T}_{A B}=0$, and this is guaranteed to happen provided that $\nabla^{\mu} T_{\mu \nu}=0$.

Obviously, the equations of motion (6) reduce to Einstein equations when $f(\mathcal{R})=\mathcal{R}$. In general, for analyzing dynamics of small oscillations about a background geometry, $g_{A B}=g_{A B}^{0}$ with curvature scalar $\mathcal{R}_{0}, f(\mathcal{R})$ must be regular at $\mathcal{R}=\mathcal{R}_{0}$. In particular, as suggested by $(6), f(\mathcal{R})$ must be regular at the origin and $f(0)$ must vanish (i.e., bulk cosmological constant must vanish) for $f(\mathcal{R})$ to admit a flat background geometry.

For determining how higher curvature gravity influences interactions among the brane matter, it is necessary to determine the propagating modes which couple to the matter stress tensor. This requires expansion of the action density in (5) by using (1) up to the desired order in $h_{A B}$. The zeroth order term vanishes by $f(0)=0$ constraint whose necessity was mentioned above. The terms first order in $h_{A B}$ vanish by equations of motion. The quadratic part, on the other hand, turns out to be

$$
\begin{gathered}
S_{h}=\int d^{D} x\left[\frac{1}{2} h_{A B}(x) \mathcal{O}^{A B C D}(x) h_{C D}(x)\right. \\
\left.-\frac{1}{\bar{M}_{D}^{(D-2) / 2}} h_{A B}(x) \mathcal{T}(x)^{A B}\right]
\end{gathered}
$$

such that propagator of $h_{A B}(x)$, defined via the relation

$\mathcal{O}_{A B C D}(x) \mathcal{D}^{C D E F}\left(x, x^{\prime}\right)=\frac{1}{2} \delta^{D}\left(x-x^{\prime}\right)\left(\delta_{A}^{E} \delta_{B}^{F}+\delta_{B}^{E} \delta_{A}^{F}\right)$,

takes the form

$$
\begin{aligned}
-i \mathcal{D}^{A B C D}\left(p^{2}\right) \\
=-\left(\frac{f^{\prime}(0)+2 f^{\prime \prime}(0) p^{2}}{(D-2) f^{\prime}(0)+2(D-1) f^{\prime \prime}(0) p^{2}}\right) \\
\quad \times \frac{1}{f^{\prime}(0) p^{2}} \eta^{A B} \eta^{C D} \\
\quad+\frac{1}{2 f^{\prime}(0) p^{2}}\left(\eta^{A C} \eta^{B D}+\eta^{A D} \eta^{B C}\right) \\
\quad+\frac{2 f^{\prime \prime}(0) p^{2}}{(D-2) f^{\prime}(0)+2(D-1) f^{\prime \prime}(0) p^{2}} \frac{1}{f^{\prime}(0) p^{4}} \\
\quad \times\left(\eta^{C D} p^{A} p^{B}+\eta^{A B} p^{C} p^{D}\right)
\end{aligned}
$$




$$
\begin{aligned}
& +\frac{(\xi-1)}{2 f^{\prime}(0) p^{4}}\left(\eta^{B D} p^{A} p^{C}+\eta^{D A} p^{C} p^{B}+\eta^{A C} p^{B} p^{D}\right. \\
& \left.+\eta^{C B} p^{D} p^{A}\right) \\
& +\frac{2(D-2) f^{\prime \prime}(0) p^{2}}{(D-2) f^{\prime}(0)+2(D-1) f^{\prime \prime}(0) p^{2}} \\
& \times \frac{1}{f^{\prime}(0) p^{6}} p^{A} p^{B} p^{C} p^{D}
\end{aligned}
$$

in momentum space. It is clear that

$$
f^{\prime}(0)>0
$$

as otherwise all graviton modes become ghost. Therefore, if one is to prevent ghosty modes participating in physical processes it is necessary to keep $f^{\prime}(0)$ positive definite. The parameter $\xi$ in (10) arises from the gauge fixing term

$\mathcal{L}_{g}=\frac{f^{\prime}(0)}{\xi} \eta^{A C}\left(\partial^{B} h_{A B}-\frac{1}{2} \partial_{A} h_{B}^{B}\right)\left(\partial^{D} h_{C D}-\frac{1}{2} \partial_{C} h_{D}^{D}\right)$

added to the $h_{A B}$ action density in (8). Here, $f^{\prime}(0)$ is introduced to match the terms generated by $\mathcal{L}_{g}$ with the ones in (8). The propagator (10) depends explicitly on the second derivative of $f(\mathcal{R})$ evaluated at the origin, and it correctly reduces to the graviton propagator in Einstein gravity $[8,9]$ when $f^{\prime \prime}(0)=0$ and $f^{\prime}(0)=1$. The specific choice $\xi=1$ corresponds to de Donder gauge frequently employed in quantum gravity. Obviously, one can probe $f(\mathcal{R})$ with higher and higher precision by computing higher and higher order $h_{A B}$ correlators. Indeed, for probing $f^{\prime \prime \prime \prime}(0)$, for instance, it is necessary to expand the action density in (5) up to quartic order so as to compute the requisite four-point function. Rather generically, higher the order of correlators higher the dimensions of the operators they induce. The propagator (10) induces a dimension-8 operator via graviton exchange between two matter stress tensors $[8,12]$. On the other hand, four-point function induces a dimension-16 operator via graviton exchange among four matter stress tensors.

The scattering processes which proceed with graviton exchange do exhibit new features as one switch from ADD setup to $f(\mathcal{R})$ gravity. Indeed, single graviton exchange influences various processes including $2 \rightarrow 2$ scatterings, particle selfenergies, box diagrams and as such. The tree level processes are sensitive to virtual states associated with the propagation of graviton in the bulk. On the other hand, loop level processes involve particle virtualities both on the brane and in the bulk. In this sense, tree level processes offer some degree of simplicity and clarity for disentangling the graviton contribution (see [12] for a through analysis of the virtual graviton exchange effects) from those of the SM states. Hence, in the following, we will restrict our discussions exclusively to tree level processes.

By imposing compactness of the extra space and taking its shape to be a torus as in the ADD mechanism one finds

$$
\begin{aligned}
\mathcal{T}_{A B}(x)= & \sum_{n_{1}=-\infty}^{+\infty} \ldots \sum_{n_{\delta}=-\infty}^{+\infty} \int \frac{d^{4} p}{(2 \pi)^{4}} \frac{1}{\sqrt{V_{\delta}}} \\
& \times e^{-i\left(k \cdot z-\frac{\vec{n} \cdot \vec{y}}{R}\right)} \delta_{A}^{\mu} \delta_{B}^{\nu} T_{\mu \nu}(k),
\end{aligned}
$$

where $\left(n_{1}, \ldots, n_{\delta}\right)$ is a $\delta$-tuple of integers. Given this Fourier decomposition of the stress tensor, the amplitude for an onbrane system $a$ to make a transition into another on-brane system $b$ becomes

$\mathcal{A}\left(k^{2}\right)=\frac{1}{\bar{M}_{\mathrm{Pl}}^{2}} \sum_{\vec{n}} T_{\mu \nu}^{(a)}(k) \mathcal{D}^{\mu \nu \lambda \rho}\left(k^{2}-\frac{\vec{n} \cdot \vec{n}}{R^{2}}\right) T_{\lambda \rho}^{(b)}(k)$,

where use has been made of (3) in obtaining $1 / \bar{M}_{\mathrm{Pl}}^{2}$ factor in front. Though we are dealing with a tree-level process the amplitude involves a summation over all Kaluza-Klein levels due to the fact that these states are inherently virtual because of their propagation off the brane. Conservation of energy and momentum implies that only the first two terms in the propagator (10) contributes to (14). Therefore, after performing summation the transition amplitude (14) takes the form

$$
\begin{aligned}
\mathcal{A}\left(k^{2}\right)= & \frac{S_{\delta-1}}{(2 \pi)^{\delta}} \frac{1}{\bar{M}_{D}^{4} f^{\prime}(0)}\left(\frac{\Lambda}{\bar{M}_{D}}\right)^{\delta-2} R\left(\frac{\Lambda}{\sqrt{k^{2}}}\right) \\
& \times\left(T_{\mu \nu}^{(a)} T^{(b) \mu \nu}-\frac{1}{\delta+2} T_{\mu}^{(a) \mu} T_{v}^{(b) v}\right) \\
& +\frac{(\delta+4)}{2(\delta+2)(\delta+3)} \frac{S_{\delta-1}}{(2 \pi)^{\delta}} \frac{1}{\bar{M}_{D}^{4} f^{\prime}(0)}\left(\frac{\Lambda}{\bar{M}_{D}}\right)^{\delta-2} \\
& \times R\left(\frac{\Lambda}{\sqrt{\tilde{k}^{2}}}\right) T_{\mu}^{(a) \mu} T_{v}^{(b) v}
\end{aligned}
$$

which exhibits a huge enhancement $\mathcal{O}\left(\bar{M}_{\mathrm{Pl}}^{2} / \bar{M}_{D}^{2}\right)$ compared to (14) due to the contributions of finely-spaced Kaluza-Klein levels [5]. Here $S_{\delta-1}=\left(2 \pi^{\delta / 2}\right) / \Gamma(\delta / 2)$ is the surface area of $\delta$-dimensional unit sphere and $\Lambda$ (which is expected to be $\mathcal{O}\left(\bar{M}_{D}\right)$ since above $\bar{M}_{D}$ underlying quantum theory of gravity completes the classical treatment pursued here) is the ultraviolet cutoff needed to tame divergent summation over Kaluza-Klein levels. In fact, $\mathcal{A}\left(k^{2}\right)$ exhibits a strong dependence on $\Lambda$, as suggested by (see also series expressions of $R\left(\Lambda / \sqrt{k^{2}}\right)$ derived in $[8,9])$

$$
\begin{aligned}
R\left(\frac{\Lambda}{\sqrt{k^{2}}}\right)= & -i \frac{\pi}{2}\left(\frac{k^{2}}{\Lambda^{2}}\right)^{\frac{\delta}{2}-1}+\frac{\pi}{2}\left(\frac{k^{2}}{\Lambda^{2}}\right)^{\frac{\delta}{2}-1} \cot \frac{\pi \delta}{2} \\
& -\frac{1}{\delta-2} 2 F_{1}\left(1,1-\frac{\delta}{2}, 2-\frac{\delta}{2}, \frac{k^{2}}{\Lambda^{2}}\right)
\end{aligned}
$$

for $0 \leqslant k^{2} \leqslant \Lambda^{2}$, and

$$
R\left(\frac{\Lambda}{\sqrt{k^{2}}}\right)=\frac{1}{\delta} \frac{\Lambda^{2}}{k^{2}}{ }_{2} F_{1}\left(1, \frac{\delta}{2}, 1+\frac{\delta}{2}, \frac{\Lambda^{2}}{k^{2}}\right)
$$

for $k^{2}<0$ or $k^{2}>\Lambda^{2}$. The imaginary part of $R$, relevant for the timelike propagator (16), is generated by exchange of onshell gravitons, i.e., those Kaluza-Klein levels satisfying $k^{2}=$ $\vec{n} \cdot \vec{n} / R^{2}$. On the other hand, its real part follows from exchange of off-shell gravitons. For spacelike propagator, the scattering amplitude (17) is real since in this channel Kaluza-Klein levels cannot come on shell.

The first and second lines of $\mathcal{A}\left(k^{2}\right)$ in (15), except for the overall $1 / f^{\prime}(0)$ factor in front, are identical to the single graviton exchange amplitude computed within the ADD setup $[8,9]$. 
In fact, operators $T_{\mu \nu}^{(a)} T^{(b) \mu \nu}$ and $T_{\mu}^{(a) \mu} T_{\nu}^{(b) v}$ are collectively induced by exchange of $J=2$ and $J=0$ modes of gravity waves $h_{A B}[8,9]$. The third and fourth lines at right-hand side, on the other hand, are a completely new contribution not found in ADD setup. The structure of the induced interaction, $T_{\mu}^{(a) \mu} T_{\nu}^{(b) \nu}$, implies that it is induced by exchange of a scalar field, different than the graviscalar which induces the same type operator in the first and second lines of (15). The sources of this additional interaction is nothing but the scalar field $\phi$ defined in (4). Therefore, the main novelty in $\mathcal{A}\left(k^{2}\right)$ lies in the third and fourth lines at right-hand side of (15) which is recognized to be generated by the exchange of a scalar field with non-vanishing bare mass-squared

$m_{\phi}^{2}=-\frac{\delta+2}{2(\delta+3)} \frac{f^{\prime}(0)}{f^{\prime \prime}(0)}$

so that $\tilde{k}^{2}=k^{2}-m_{\phi}^{2}$ in (15). The nature of the scalar field $\phi$ depends on sign of $f^{\prime \prime}(0)$ : $\phi$ is a real scalar for $f^{\prime \prime}(0)<0$ and a tachyon for $f^{\prime \prime}(0)>0$. Moreover, when $f^{\prime \prime}(0)=0$ it is clear that $f(\mathcal{R})$ gravity remains Einsteinian up to $\mathcal{O}\left(\mathcal{R}^{3}\right)$ and this reflects itself by decoupling of $\phi$ from propagator (10) and transition amplitude (14) since now $\phi$ is an infinitely massive scalar. On the other hand, when $f^{\prime \prime}(\mathcal{R})$ is singular at the origin the bare mass of $\phi$ vanishes and thus $\mathcal{A}\left(k^{2}\right)$ simplifies to the first and second lines of (15) such that coefficient of $T_{\mu}^{(a) \mu} T_{v}^{(b) v}$ changes from $-1 /(\delta+2)$ to $-1 /(2(\delta+3))$. A tachyonic scalar, $m_{\phi}^{2}<0$, decouples from the transition amplitude (15) as $k^{2}-$ $m_{\phi}^{2} \rightarrow \infty$. This can be seen from the asymptotic behavior of (16) by noting that on-shell graviton exchange is shut off for $k^{2}-m_{\phi}^{2} \geqslant \Lambda^{2}$. Similarly, a true scalar, $m_{\phi}^{2}>0$, also decouples from the transition amplitude (15) when $k^{2}-m_{\phi}^{2} \rightarrow-\infty$ as suggested by the asymptotic behavior (17). In the next section we will study higher dimensional operators induced by $f(\mathcal{R})$ gravity and their collider signatures.

\section{Higher dimensional operators from $f(\mathcal{R})$ gravity}

The impact of $f(\mathcal{R})$ gravity on the transition amplitude (15) is restricted to occur via the dimension- 8 operator $T_{\mu}^{(a) \mu} T_{\nu}^{(b) v}$. This operator involves traces of the stress tensors of both systems $a$ and $b$. In general, trace of the energy-momentum tensor, at tree level, is directly related to the sources of conformal breaking in the system [13]. It may be instructive to determine stress tensors and their traces for fundamental fields. The energy and momentum of a massive vector field $A_{\mu}$ is contained in the conserved stress tensor

$$
\begin{aligned}
T_{\mu \nu}^{(J=1)}= & \eta_{\mu \nu}\left(\frac{1}{4} F^{\lambda \rho} F_{\lambda \rho}-\frac{1}{2} M_{A}^{2} A_{\lambda} A^{\lambda}\right) \\
& -\left(F_{\mu}^{\rho} F_{\nu \rho}-M_{A}^{2} A_{\mu} A_{\nu}\right)
\end{aligned}
$$

whose trace

$T_{\mu}^{(J=1) \mu}=-M_{A}^{2} A_{\mu} A^{\mu}$

demonstrates that vector boson mass breaks conformal invariance explicitly. On the other hand, conserved energy- momentum tensor for a massive fermion reads as

$$
\begin{aligned}
T_{\mu \nu}^{(J=1 / 2)}= & -\eta_{\mu \nu}\left(\bar{\psi} i \not \partial \psi-m_{\psi} \bar{\psi} \psi\right)+\frac{i}{2} \bar{\psi}\left(\gamma_{\mu} \partial_{\nu}+\gamma_{\nu} \partial_{\mu}\right) \psi \\
& +\frac{1}{4}\left[2 \eta_{\mu \nu} \partial^{\lambda}\left(\bar{\psi} i \gamma_{\lambda} \psi\right)-\partial_{\mu}\left(\bar{\psi} i \gamma_{\nu} \psi\right)\right. \\
& \left.-\partial_{\nu}\left(\bar{\psi} i \gamma_{\mu} \psi\right)\right]
\end{aligned}
$$

whose trace

$T_{\mu}^{(J=1 / 2) \mu}=m_{\psi} \bar{\psi} \psi$

shows that fermion mass breaks conformal invariance explicitly. In contrast to vector fields and spinors, trace of the stress tensor for a scalar field is not directly related to its mass term. In fact, $T_{\mu}^{(J=0) \mu}$ is non-zero even for a massless scalar. For a scalar field $\Phi$ to have $T_{\mu}^{(J=0) \mu}$ to be proportional to its mass term it is necessary to introduce gauging $\square \Phi \rightarrow\left(\square-\zeta_{c} \mathcal{R}\right) \Phi$ with 'gauge coupling' $\zeta_{c}=(D-2) /(4(D-1))$ [14]. The curvature scalar serves as the gauge field of local scale invariance. This gauging gives rise to additional terms in the stress tensor of $\Phi$, and they do not vanish even in the flat limit. More explicitly, for a massive complex scalar with quartic coupling the stress tensor reads as

$$
\begin{aligned}
T_{\mu \nu}^{(J=0)}= & -\eta_{\mu \nu}\left[\partial^{\rho} \Phi^{\dagger} \partial_{\rho} \Phi-M_{\Phi}^{2} \Phi^{\dagger} \Phi-\lambda\left(\Phi^{\dagger} \Phi\right)^{2}\right] \\
& +\partial_{\mu} \Phi^{\dagger} \partial_{\nu} \Phi+\partial_{\nu} \Phi^{\dagger} \partial_{\mu} \Phi+2 \zeta\left(\eta_{\mu \nu} \square-\partial_{\mu} \partial_{\nu}\right) \Phi^{\dagger} \Phi
\end{aligned}
$$

whose trace

$$
\begin{aligned}
T_{\mu}^{(J=0) \mu}= & -2(1-6 \zeta)\left[\partial^{\rho} \Phi^{\dagger} \partial_{\rho} \Phi-\lambda\left(\Phi^{\dagger} \Phi\right)^{2}\right] \\
& +4(1-3 \zeta) M_{\Phi}^{2} \Phi^{\dagger} \Phi
\end{aligned}
$$

reduces to $T_{\mu}^{(J=0) \mu}=2 M_{\Phi}^{2} \Phi^{\dagger} \Phi$ for $\zeta=\zeta_{c} \equiv 1 / 6$, as desired. For $\zeta \neq \zeta_{c}$, say $\zeta=0, T_{\mu}^{(J=0) \mu}$ involves kinetic term, selfinteraction potential $\lambda\left(\Phi^{\dagger} \Phi\right)^{2}$ as well as mass term of the scalar field. The terms proportional to $\zeta$ in (23) might be regarded as either following from coupling of $\Phi$ to curvature scalar as discussed above, or as a field-theoretic technicality to improve properties of the dilatation current [15].

The stress tensor traces (20), (22) and (24) with $\zeta=$ $1 / 6$ show that effects of graviscalar exchange (the operator $T_{\mu}^{(a) \mu} T_{\nu}^{(b) v}$ in the first and second lines of $\left.(15)\right)$ and $f(\mathcal{R})$ gravity (the operator in the third and fourth lines of (15)) can show up only in those scattering processes which involve massive brane matter at their initial and final states. Their phenomenological viability depends on how heavy the brane states compared to $\bar{M}_{D}$. For instance, high-energy processes initiated by $e^{+} e^{-}$annihilation or $\gamma \gamma$ scattering or $p \bar{p}$ annihilation cannot probe the operator $T_{\mu}^{(a) \mu} T_{\nu}^{(b) v}$ in (15). On the other hand, scattering processes which involve heavy fermions (e.g., bottom and top quarks, muon and tau lepton), weak bosons $W^{ \pm}$, $Z$, and Higgs boson $h$ are particularly useful for probing the gravitational effects. Each of these processes provides an arena for probing effects of scalar graviton exchange in general, and $f(\mathcal{R})$ gravity effects in particular. It might be instructive to depict explicitly how $\mathcal{A}\left(k^{2}\right)$ differs from that computed within the ADD setup by a number of specific scattering processes. 
Concerning $2 \rightarrow 2$ scattering of weak bosons one can consider, for instance, the process $Z_{\alpha}\left(p_{1}\right) Z_{\beta}\left(p_{2}\right) \rightarrow Z_{\gamma}\left(k_{1}\right) Z_{\lambda}\left(k_{2}\right)$ which is described by the amplitude

$$
\begin{aligned}
\mathcal{A}_{Z Z} & \rightarrow Z Z\left(k^{2}\right) \\
= & \mathcal{A}_{\mathrm{SM}}\left(k^{2}\right)+\frac{1}{f^{\prime}(0)} \mathcal{A}_{\mathrm{ADD}}\left(k^{2}\right) \\
& +\frac{(\delta+4)}{2(\delta+2)(\delta+3)} \frac{S_{\delta-1}}{(2 \pi)^{\delta}} \frac{M_{Z}^{4}}{\bar{M}_{D}^{4} f^{\prime}(0)}\left(\frac{\Lambda}{\bar{M}_{D}}\right)^{\delta-2} \\
& \times\left\{R\left(\frac{\Lambda}{\sqrt{\tilde{s}}}\right) \eta_{\alpha \beta} \eta_{\gamma \lambda}+R\left(\frac{\Lambda}{\sqrt{\tilde{t}}}\right) \eta_{\alpha \gamma} \eta_{\beta \lambda}\right. \\
& \left.+R\left(\frac{\Lambda}{\sqrt{\tilde{u}}}\right) \eta_{\alpha \lambda} \eta_{\beta \gamma}\right\} \\
& \times \epsilon_{Z}^{\alpha}\left(p_{1}\right) \epsilon_{Z}^{\beta}\left(p_{2}\right) \epsilon_{Z}^{\star \gamma}\left(k_{1}\right) \epsilon_{Z}^{\star \lambda}\left(k_{2}\right)
\end{aligned}
$$

after using (19) in (15). In this expression, $s=\left(p_{1}+p_{2}\right)^{2}=$ $\left(k_{1}+k_{2}\right)^{2}, t=\left(k_{1}-p_{1}\right)^{2}$ and $u=\left(k_{2}-p_{1}\right)^{2}=4 M_{Z}^{2}-s-t$ are Mandelstam variables, and $\epsilon_{Z}^{\mu}$ stands for the polarization vector of $Z$ boson. The amplitudes $\mathcal{A}_{\mathrm{SM}}\left(k^{2}\right)$ and $\mathcal{A}_{\mathrm{ADD}}\left(k^{2}\right)$ can be found in [16]. Obviously, $f(\mathcal{R})$ gravity effects get pronounced when $M_{D}$ lies close to $M_{Z}$. Clearly, $\sigma(Z Z \rightarrow Z Z)$ feels $f(\mathcal{R})$ gravity via square of the third term in (25) and its interference with SM and ADD contributions.

The fermion scattering $\psi_{1}\left(p_{1}\right) \psi_{1}\left(p_{2}\right) \rightarrow \psi_{2}\left(k_{1}\right) \psi_{2}\left(k_{2}\right)$ is described by

$$
\begin{aligned}
\mathcal{A}_{\psi_{1}} \psi_{1} \rightarrow \psi_{2} \psi_{2} & \left(k^{2}\right) \\
= & \mathcal{A}_{\mathrm{SM}}\left(k^{2}\right)+\frac{1}{f^{\prime}(0)} \mathcal{A}_{\mathrm{ADD}}\left(k^{2}\right) \\
& +\frac{(\delta+4)}{2(\delta+2)(\delta+3)} \frac{S_{\delta-1}}{(2 \pi)^{\delta}} \frac{m_{\psi_{1}} m_{\psi_{2}}}{\bar{M}_{D}^{4} f^{\prime}(0)}\left(\frac{\Lambda}{\bar{M}_{D}}\right)^{\delta-2} \\
& \times R\left(\frac{\Lambda}{\sqrt{\tilde{s}}}\right) \bar{\psi}_{1}\left(p_{1}\right) \psi_{1}\left(p_{2}\right) \bar{\psi}_{2}\left(k_{1}\right) \psi_{2}\left(k_{2}\right)
\end{aligned}
$$

after using (21) in (15). If $\psi_{1}$ and $\psi_{2}$ are identical fermions then $t$ and $u$ channel contributions must also be included. The SM and ADD pieces in this amplitude can be found in [8,9]. The heavy fermion scatterings (e.g., $t t \rightarrow t t, b b \rightarrow t t, \tau \tau \rightarrow t t$ ) are potential processes for highlighting effects of $f(\mathcal{R})$ gravity.

The $2 \rightarrow 2$ scattering of Higgs bosons provides another interesting channel to probe $f(\mathcal{R})$ gravity effects. Indeed, after expanding (23) around the electroweak vacuum $\Phi=(v+$ $h, 0) / \sqrt{2}$ with $v \simeq 246 \mathrm{GeV}$, the amplitude for $h\left(p_{1}\right) h\left(p_{2}\right) \rightarrow$ $h\left(k_{1}\right) h\left(k_{2}\right)$ scattering takes the form

$$
\begin{aligned}
\mathcal{A}_{h h} \rightarrow h h & \left(k^{2}\right) \\
= & \mathcal{A}_{\mathrm{SM}}\left(k^{2}\right)+\frac{1}{f^{\prime}(0)} \mathcal{A}_{\mathrm{ADD}}\left(k^{2}\right) \\
& +\frac{(\delta+4)}{8(\delta+2)(\delta+3)} \frac{S_{\delta-1}}{(2 \pi)^{\delta}} \frac{m_{h}^{4}}{\bar{M}_{D}^{4} f^{\prime}(0)}\left(\frac{\Lambda}{\bar{M}_{D}}\right)^{\delta-2} \\
& \times\left\{R\left(\frac{\Lambda}{\sqrt{\tilde{s}}}\right)+R\left(\frac{\Lambda}{\sqrt{\tilde{t}}}\right)+R\left(\frac{\Lambda}{\sqrt{\tilde{u}}}\right)\right\},
\end{aligned}
$$

where $m_{h}^{2}=-2 M_{\Phi}^{2}$ is the Higgs boson mass-squared. It is clear that size of $f(\mathcal{R})$ gravity effects depends crucially on how close $M_{D}$ is to $m_{h}$. Calculations [17] within ADD setup show that graviton exchange can have significant impact on $h\left(p_{1}\right) h\left(p_{2}\right) \rightarrow h\left(k_{1}\right) h\left(k_{2}\right)$, and thus, resulting deviation from the SM expectation might be of observable size.

The $2 \rightarrow 2$ scattering processes mentioned above illustrate how $f(\mathcal{R})$ gravity influences certain observables to be measured in collider experiments. Beyond these, there are, of course various observables which can sense $f(\mathcal{R})$ gravity. For instance, $h Z Z$ coupling, which is crucial for Higgs boson search via Bjorken process, gets also modified by graviton exchange [18] via $T_{\mu \nu}^{(J=0)} T_{\lambda \rho}^{(J=1)}$ correlator. The discussions above show that, independent of what brane matter species are taking part in a specific process, entire novelty brought about by $f(\mathcal{R})$ gravity is contained in the third and fourth lines of (15), and thus, it proves useful to carry out a comparative analysis of this contribution with the same structure present in the ADD setup, for completeness. In fact, ratio of the coefficients of $T_{\mu}^{(a) \mu} T_{v}^{(b) v}$ in (15)

$$
Q\left(k^{2}\right)=-\frac{(\delta+4)}{2(\delta+3)} \frac{R\left(\Lambda / \sqrt{\tilde{k}^{2}}\right)}{R\left(\Lambda / \sqrt{k^{2}}\right)}
$$

is a useful quantity for such a comparative analysis. For determining how finite $f^{\prime \prime}(0)$ influences the scattering processes it suffices to determine $m_{\phi}^{2}$ dependence of $Q\left(k^{2}\right)$ for given values of $k^{2}, \delta$ and $\Lambda \sim \bar{M}_{D}$. In accord with future collider searches, one can take, for instance, $k^{2}=(1 \mathrm{TeV})^{2}$ and $\Lambda=$ $\bar{M}_{D}=5 \mathrm{TeV}$, and examine $m_{\phi}^{2}$ dependencies of $\operatorname{Re}\left[Q\left(k^{2}\right)\right]$ and $\operatorname{Im}\left[Q\left(k^{2}\right)\right]$ separately. In fact, depicted in Figs. 1 and 2 are, respectively, the variations of $\operatorname{Re}\left[Q\left(k^{2}\right)\right]$ and $\operatorname{Im}\left[Q\left(k^{2}\right)\right]$ with $m_{\phi}^{2}$. In the figures, $m_{\phi}^{2}$ varies from $-(30 \mathrm{TeV})^{2}$ up to $+(30 \mathrm{TeV})^{2}$ for each number of extra dimensions considered: $\delta=3$ (solid), $\delta=5$ (dot-dashed) and $\delta=7$ (short-dashed). As suggested by (18), positive and negative $m_{\phi}^{2}$ values in the figures correspond, respectively, to negative and positive values of $f^{\prime \prime}(0)$ since $f^{\prime}(0)$ has already been restricted to take positive values to prevent graviton becoming a ghost (see the propagator (10)). On the other hand, if $\mathcal{A}\left(k^{2}\right)$ in (15) exhibits a timelike $\left(\tilde{k}^{2}>0\right)$ or spacelike $\left(\tilde{k}^{2}<0\right)$ propagation depends on how $k^{2}$ compares with $m_{\phi}^{2}$. With the values of parameters given above, the figures illustrate cases where $k^{2}>0$ yet $\tilde{k}^{2}$ varies over a wide range of values comprising spacelike and timelike behaviors as well as a heavy $\phi$, i.e., $\left|m_{\phi}^{2}\right| \gg \Lambda^{2}$.

The overall behaviors of both figures suggest that $F(\mathcal{R})$ gravity effects fade away for large $\left|m_{\phi}^{2}\right|$, as expected. Both real and imaginary parts of $Q\left(k^{2}\right)$ exhibit a narrow peak at $m_{\phi}^{2}=-(24 \mathrm{TeV})^{2}$ which corresponds to resonating of the transition amplitude by Kaluza-Klein levels with mass-squared $=$ $k^{2}-m_{\phi}^{2}=\Lambda^{2}$. From Fig. 1 it is clear that $\operatorname{Re}\left[Q\left(k^{2}\right)\right]$ becomes significant at large $\delta$ and negative $m_{\phi}^{2}$. This is also seen to hold for $\operatorname{Im}\left[Q\left(k^{2}\right)\right]$ from Fig. 2. Obviously, $f(\mathcal{R})$ gravity predictions differ from ADD ones for moderate (with respect to scale $\Lambda$ ) negative $m_{\phi}^{2}$ or equivalently for sufficiently small and positive $f^{\prime \prime}(0)$ (see Eq. (18) for details). Indeed, for positive values of $m_{\phi}^{2}$ or equivalently for negative $f^{\prime \prime}(0)$ the strength of $f(\mathcal{R})$ gravity contribution remains significantly below the ADD one. 


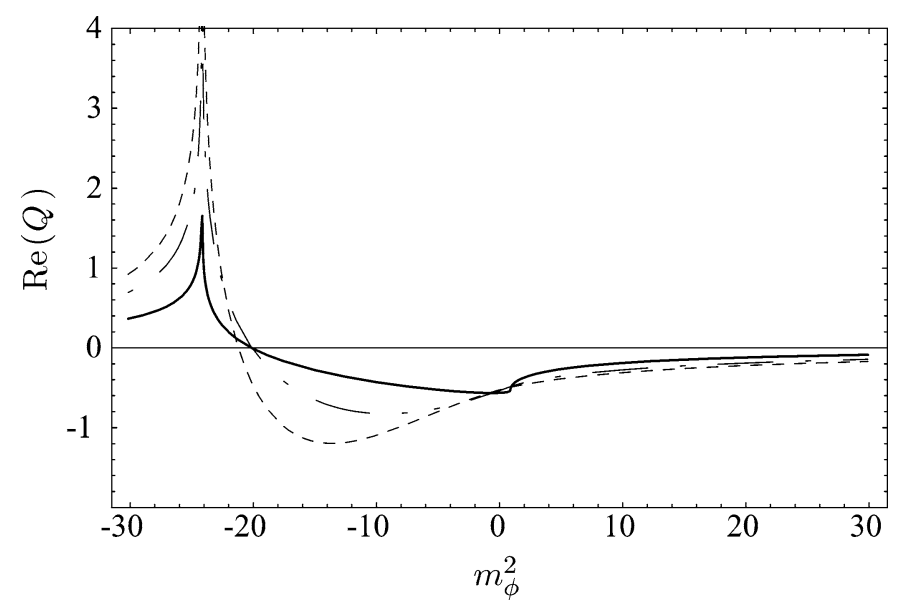

Fig. 1. The dependence of $\operatorname{Re}\left[Q\left(k^{2}\right)\right]$ on $m_{\phi}^{2}$ for $k^{2}=(1 \mathrm{TeV})^{2}, \Lambda=$ $\bar{M}_{D}=5 \mathrm{TeV}$, and $\delta=3$ (solid curve), $\delta=5$ (dot-dashed curve) and $\delta=7$ (short-dashed curve). We vary $m_{\phi}^{2}$ from $-(30 \mathrm{TeV})^{2}$ up to $+(30 \mathrm{TeV})^{2}$.

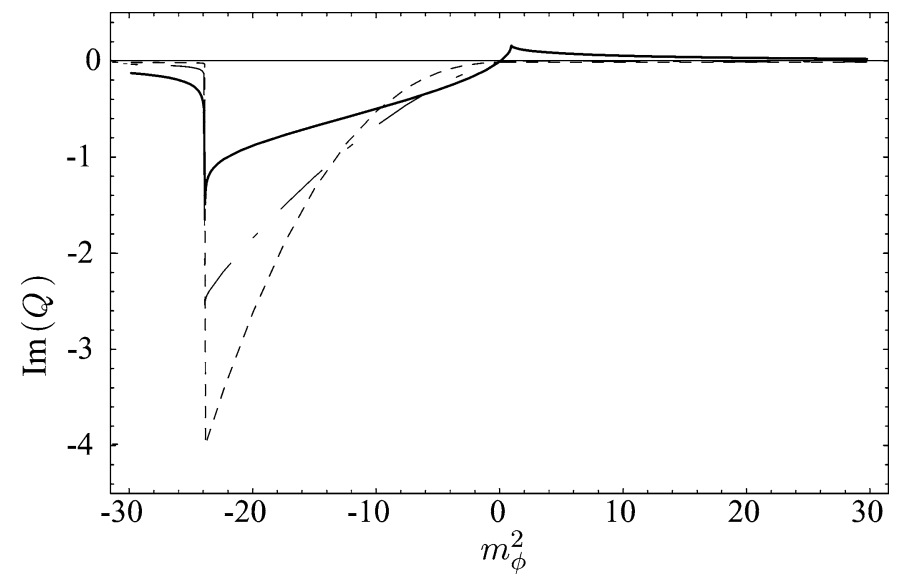

Fig. 2. The same as in Fig. 1 but for $\operatorname{Im}\left[Q\left(k^{2}\right)\right]$.

The behaviors of $\operatorname{Re}\left[Q\left(k^{2}\right)\right]$ and $\operatorname{Im}\left[Q\left(k^{2}\right)\right]$ illustrated in these figures remain qualitatively similar for other $k^{2}, \Lambda$ or $\delta$ combinations. Therefore, one concludes that generalization of ADD mechanism based on Einstein-Hilbert action density $\bar{M}_{D}^{2} \mathcal{R}$ to $\bar{M}_{D}^{2} f(\mathcal{R})$ type higher curvature gravity theory can have observable effects on high energy processes (for instance, the ones listed in (25), (26) and (27) listed above) when $f^{\prime \prime}(0)$ is positive and $\mathcal{O}\left(1 / \bar{M}_{D}^{2}\right)$.

\section{Yet more signatures of $f(\mathcal{R})$ gravity}

So far we have focussed mainly on higher dimensional operators induced by tree-level virtual graviton exchange. Clearly, effects of higher dimensional gravity on brane matter are not restricted to such processes: graviton can contribute to selfenergies, effective vertices or box diagrams of brane matter; graviton can be emitted off the brane matter; and graviton can decay into brane matter. In this section we will discuss such processes briefly for illustrating how $f(\mathcal{R})$ gravity effects differ from those found in the ADD setup.

First of all, as suggested by (5), couplings of the gravity waves $h_{A B}(x)$ to brane matter are identical in ADD and $f(\mathcal{R})$ gravity setups. Therefore, distinction between the two frameworks rests mainly on the additional scalar field (4) imbrued in the $f(\mathcal{R})$ gravity dynamics. Consequently, detection of $f(\mathcal{R})$ gravity effects requires scattering processes on the brane to be sensitive to the new energy threshold $m_{\phi}$ not found in the ADD setup.

Let us consider first role of $f(\mathcal{R})$ gravity on brane-localized loops. The simplest of such processes is the self-energy of a brane particle. One may consider, for instance, self-energy of the $Z$ boson (or any of the massive SM fields mentioned in the last section). At the level of a single graviton exchange one finds

$$
\begin{aligned}
-i \Pi(q) & \\
= & -i \Pi_{\mathrm{SM}}(q)-i \frac{1}{f^{\prime}(0)} \Pi_{\mathrm{ADD}}(q)-i \Pi_{\text {seagull }}\left(q^{2}\right) \\
& -\frac{M_{Z}^{4}}{\bar{M}_{\mathrm{Pl}}^{2} f^{\prime}(0)} \frac{\delta+4}{(\delta+2)(\delta+3)} \\
& \times \sum_{\vec{n}} \int \frac{d^{4} k}{(2 \pi)^{4}} \frac{1}{k^{2}-m_{\phi}^{2}-\frac{\vec{n} \cdot \vec{n}}{R^{2}}+i \epsilon} \frac{1}{(q+k)^{2}-M_{Z}^{2}+i \epsilon},
\end{aligned}
$$

where contribution of the four-point vertex that binds gravitons and $Z$ bosons is contained in the seagull contribution. The summation and integration involved in this expression are difficult to evaluate analytically, and therefore, one may eventually need to resort some numerical techniques [9]. However, at least for vanishing external momentum, one can show that $f(\mathcal{R})$ gravity contribution in the second and third lines of (29) is diminished at large $\left|m_{\phi}\right|^{2}$, and is particularly pronounced when $\left|m_{\phi}\right|^{2} \sim M_{Z}^{2}$ and $m_{\phi}^{2}<0$. Therefore, when $f^{\prime \prime}(0) \sim 1 / M_{Z}^{2}$ one expects observable enhancements in the $Z$ boson self-energy (see [19] and [9] for analyses of the Higgs boson self-energy).

Having discussed effects of $f(\mathcal{R})$ gravity on brane-localized loops we now turn to an analysis of production and decays of the graviton. In these processes graviton is a physical particle described by asymptotically free states connected by the $S$-matrix elements. Therefore, the scalar field $\phi$ imbrued in $f(\mathcal{R})$ dynamics must be endowed with a positive mass-squared for its decays and productions to be observable. Consequently, $f(\mathcal{R})$ gravity effects on graviton production and decay exist within $m_{\phi}^{2}>0$ domain. However, as suggested by Figs. 1 and 2, $f(\mathcal{R})$ gravity contribution, the second line of (15), stays significantly below the corresponding contribution in ADD setup. This implies, in particular, that production and decay of $\phi$ graviton are suppressed relative to those of the $J=2$ and $J=0$ gravitons.

The above observation is confirmed by the fact that when the looping particles come on their mass shells, as dictated by the optical theorem, the $Z$ boson self-energy (29) above represents the Drell-Yan production of graviton and $Z$ boson at lepton (via $e^{+} e^{-} \rightarrow Z^{\star} \rightarrow$ graviton $+Z$ annihilation) or hadron (via $q \bar{q} \rightarrow Z^{\star} \rightarrow$ graviton $+Z$ annihilation) colliders. The main novelty brought about by $f(\mathcal{R})$ gravity is the production of $\phi$ (in addition to $J=0$ and $J=2$ gravitons) when the center-of-mass energy of the collider is sufficiently large, i.e., $s \geqslant m_{\phi}^{2}+M_{Z}^{2}$. This phenomenon reflects itself by a sudden change in the number of events (similar to opening of 
$W^{+} W^{-}$channel at LEP experiments). The dominant contribution to graviton emission comes from Kaluza-Klein levels in the vicinity of $R^{2}\left(M_{Z}^{2}-m_{\phi}^{2}\right)$. The emission of gravitons from the brane is not restricted to such $2 \rightarrow 2$ processes, however. Indeed, massive brane-localized states can decay into gravitons, including $\phi$ itself, and this reflects itself as an increase in their invisible widths (see, for instance, [19] for a detailed discussion of the Higgs boson width).

There are, of course, inverse processes to graviton emission. Indeed, gravitons propagating in the bulk can decay into brane matter when they land on the brane. The graviton decay channels can open only if their Kaluza-Klein level is high enough [9]. The only exception to this is the $\phi$ graviton which can decay into brane matter even at zeroth Kaluza-Klein level provided that its mass, $m_{\phi}$, is larger than those of the daughter particles. Detailed discussions of the production and decays of gravitons (as well as those of the right-handed neutrinos propagating in the bulk [20]) in the framework of ADD mechanism can be found in [5].

This section is intended to provide a brief summary of what impact $f(\mathcal{R})$ gravity can have on processes involving braneloops, missing energy signals in brane matter scatterings, and population of brane via the graviton decays. These processes are of great importance for both collider [8,9] and cosmological $[5,20]$ purposes, and discussions provided in this section is far from being sufficient for a proper description of what effects $f(\mathcal{R})$ gravity can leave on them. From this brief analysis, combined with results of the previous section, one concludes that $f(\mathcal{R})$ gravity effects on decays and emissions of graviton cannot compete with the ADD expectations.

\section{Conclusion}

In this work we have discussed a number of phenomenological implications of $f(\mathcal{R})$ gravity in higher dimensional spacetimes with large extra spatial dimensions. In Section 2 we have derived graviton propagator about flat Minkowski background (which requires $f(0)$, i.e., cosmological constant to vanish), and have determined how it influences interactions among the brane matter. In Section 3 we have listed down a set of higher dimensional operators which exhibit an enhanced sensitivity to $f(\mathcal{R})$ gravity (compared to those operators involving light fermions or massless gauge fields). Finally, in this section we have performed a comparative study of $\mathrm{ADD}$ and $f(\mathcal{R})$ gravity predictions and determined ranges of parameters where the latter dominates over the former. The analysis therein suggests that $f(\mathcal{R})$ gravity theories with finite and positive $f^{\prime \prime}(0)$ induce potentially important effects testable at future collider studies. In Section 4 we have discussed briefly how $f(\mathcal{R})$ gravity influences loop processes on the brane as well as decays and productions of gravitons.

The analysis in this work can be applied to various laboratory, astrophysical and cosmological observables (see [5] for a detailed discussion of major observables) for examining nonEinsteinian forms of general relativity in higher dimensions. The discussions presented here are far from being complete in their coverage and phenomenological investigations. The rule of thumb to be kept in mind is that higher curvature gravity influences scatterings of massive (sufficiently heavy compared to the fundamental scale of gravity) brane matter.

\section{Acknowledgements}

The work of D.A.D. was partially supported by Turkish Academy of Sciences through GEBIP grant, and by the Scientific and Technical Research Council of Turkey through project $104 \mathrm{~T} 503$.

\section{References}

[1] E. Witten, Nucl. Phys. B 471 (1996) 135, hep-th/9602070.

[2] P. Horava, E. Witten, Nucl. Phys. B 460 (1996) 506, hep-th/9510209; P. Horava, E. Witten, Nucl. Phys. B 475 (1996) 94, hep-th/9603142.

[3] I. Antoniadis, Phys. Lett. B 246 (1990) 377.

[4] J.D. Lykken, Phys. Rev. D 54 (1996) 3693, hep-th/9603133.

[5] N. Arkani-Hamed, S. Dimopoulos, G.R. Dvali, Phys. Lett. B 429 (1998) 263, hep-ph/9803315;

N. Arkani-Hamed, S. Dimopoulos, G.R. Dvali, Phys. Rev. D 59 (1999) 086004, hep-ph/9807344.

[6] J.C. Long, H.W. Chan, J.C. Price, Nucl. Phys. B 539 (1999) 23, hep$\mathrm{ph} / 9805217$;

J.C. Long, J.C. Price, C. R. Physique 4 (2003) 337, hep-ph/0303057.

[7] V.A. Rubakov, M.E. Shaposhnikov, Phys. Lett. B 125 (1983) 136.

[8] G.F. Giudice, R. Rattazzi, J.D. Wells, Nucl. Phys. B 544 (1999) 3, hep$\mathrm{ph} / 9811291$.

[9] T. Han, J.D. Lykken, R.J. Zhang, Phys. Rev. D 59 (1999) 105006, hep$\mathrm{ph} / 9811350$.

[10] J.D. Barrow, S. Cotsakis, Phys. Lett. B 214 (1988) 515; K.I. Maeda, Phys. Rev. D 39 (1989) 3159;

S. Kalara, N. Kaloper, K.A. Olive, Nucl. Phys. B 341 (1990) 252;

G. Magnano, L.M. Sokolowski, Phys. Rev. D 50 (1994) 5039, grqc/9312008.

[11] C. Brans, H. Dicke, Phys. Rev. 124 (1961) 925.

[12] G.F. Giudice, A. Strumia, Nucl. Phys. B 663 (2003) 377, hep-ph/0301232.

[13] D.J. Gross, J. Wess, Phys. Rev. D 2 (1970) 753; J.D. Bekenstein, A. Meisels, Phys. Rev. D 22 (1980) 1313; J. Polchinski, Nucl. Phys. B 303 (1988) 226.

[14] A. Iorio, L. O'Raifeartaigh, I. Sachs, C. Wiesendanger, Nucl. Phys. B 495 (1997) 433, hep-th/9607110;

D.A. Demir, Phys. Lett. B 584 (2004) 133, hep-ph/0401163.

[15] C.G. Callan, S.R. Coleman, R. Jackiw, Ann. Phys. 59 (1970) 42.

[16] D. Atwood, S. Bar-Shalom, A. Soni, Phys. Rev. D 61 (2000) 054003, hep$\mathrm{ph} / 9906400$.

[17] X.G. He, Phys. Rev. D 61 (2000) 036007, hep-ph/9905500.

[18] D. Choudhury, A. Datta, K. Huitu, Nucl. Phys. B 673 (2003) 385, hep$\mathrm{ph} / 0302141$.

[19] G.F. Giudice, R. Rattazzi, J.D. Wells, Nucl. Phys. B 595 (2001) 250, hep$\mathrm{ph} / 0002178$.

[20] D.A. Demir, T. Gherghetta, K.A. Olive, Phys. Lett. B 549 (2002) 194, hep-ph/0209003. 